\title{
A SUFFICIENT CONDITION FOR KRALL'S CRITERION AND ITS MODIFICATIONS
}

\section{WU LINXIANG}

Department of Geophysics, Peking University

United Laboratory for Optical Astronomy, Chinese Academy of Sciences

ABSTRACT In order to determine the sense of the photospheric transverse magnetic field measured with a magnetograph, it is shown that: (1) If the magnetic lines of force through the photospheric plane are both divergent and convex upward, Krall's criterion

$$
\mathbf{B}_{\perp} \cdot \nabla B_{z}<0,
$$

is true for the transverse field $\mathbf{B}_{\perp}$ much more strongly than for the longitudinal field $B_{z}$ and the inequality in general becomes

$$
\mathbf{B}_{\perp} \cdot \nabla B_{z}-B_{z} \nabla \cdot \mathbf{B}_{\perp}<0 .
$$

(2) If the magnetic lines of force are only divergent upward, we have instead the inequality

$$
B_{z}\left[\mathrm{~B}_{\perp} \cdot \nabla\left(B_{\perp}^{2}+2 B_{z}^{2}\right)-2 B_{z}^{2} \nabla \cdot \mathrm{B}_{\perp}\right]<0
$$

for a force-free field.

\section{INTRODUCTION}

It is well known that there is a $180^{\circ}$ ambiguity in the direction of the photospheric transverse magnetic field $\mathbf{B}_{\perp}$ measured with a vector magnetograph. In order to fix the sense of the vector $\mathbf{B}_{\perp}$, Krall et al. (1982) assumed a criterion of discrimination

$$
\mathbf{B}_{\perp} \cdot \nabla B_{z}<0
$$

i.e., the angle made by the vector $\mathbf{B}_{\perp}$ with the gradient vector of the photospheric longitudinal magnetic field $B_{z}$ should be obtuse. In spite of its successful application, this assumption does not hold unconditionally, as shown by many counterevidences ( $\mathrm{Wu} \& \mathrm{Ai} 1990$ ). In the present work, we investigate the conditions under which the inequality (1) is valid and try to find more general criteria to determine the sense of $\mathbf{B}_{\perp}$. 


\section{PRELIMINARIES}

(a) If the magnitude $B$ of the magnetic field $\mathbf{B}$ decreases along the direction of the field, the cross section of the magnetic flux tube increases and the magnetic lines of force are divergent. If $B$ increases along the field, the tube cross section decreases and the lines of force are convergent. Thus, the divergence or convergence of the magnetic field lines can be expressed by the inequality

$$
\mathrm{B}_{\circ} \cdot \nabla B<0 \text { or }>0 \text {, }
$$

$B_{0}$ being the unit vector along magnetic field. Consider the magnetic field lines through the photospheric plane being divergent upward, i.e., the magnetic flux tube becomes wider as it threads through the photospheric plane from the lower side to the upper. The magnetic lines are divergent for $B_{z}>0$ and convergent for $B_{z}<0$. Hence

$$
B_{z} \mathbf{B}_{\circ} \cdot \nabla B<0
$$

on the photospheric plane wherever the lines are divergent upward.

(b) The curvature vector of a magnetic line of force can be expressed as the spatial rate of change of the unit vector $B_{0}$ in the direction of magnetic field $\mathbf{B}_{\circ} \cdot \nabla \mathbf{B}_{\circ}$. If the magnetic line of force through the photospheric plane is convex upward, the angle between the curvature vector and the unit vector $\mathbf{Z}_{\text {。 }}$ perpendicular to the photospheric plane and pointed upward is obtuse, i.e.,

$$
\mathbf{Z}_{\circ} \cdot\left(\mathbf{B}_{\circ} \cdot \nabla \mathbf{B}_{\circ}\right)<0
$$

or

$$
\mathbf{B}_{\circ} \cdot \nabla\left(\mathbf{Z}_{\circ} \cdot \mathbf{B}_{\circ}\right)=\mathbf{B}_{\circ} \cdot \nabla \frac{B_{z}}{B}<0 .
$$

Simplifying the gradient of the quotient, we have

$$
\mathrm{B} \cdot \nabla B_{z}-B_{z} \mathbf{B}_{\circ} \cdot \nabla B<0 \text {. }
$$

\section{A SUFFICIENT CONDITION}

If the magnetic lines of force through the photospheric plane are both divergent and convex upward, inequalities (2) and (3) apply to the photospheric plane simultaneously and lead directly to

$$
\mathbf{B} \cdot \nabla B_{z}<0 \text {. }
$$

To compare with inequality (1), B in (4) may be decomposed into two parts

$$
\mathbf{B}=\mathbf{B}_{\perp}+\mathbf{Z}_{\odot} B_{z} \text {. }
$$

With $Z_{\circ} \cdot \nabla=\partial / \partial z$, inequality (4) becomes

$$
\mathbf{B}_{\perp} \cdot \nabla B_{z}+B_{z} \frac{\partial B_{z}}{\partial z}<0
$$

If the second term on the left hand side is negligible in comparison with the first term, (5) is Krall's criterion (1). Such cases take place for the transverse field much stronger than the longitudinal field (for example, in the neighborhood of a neutral line) or for the transverse gradient of $B_{z}$ much stronger than the vertical gradient of $B_{z}$. Additionally, (1) can also be deduced for the rare case $\partial B_{z}^{2} / \partial z>0$, i.e., the intensity of the longitudinal field increasing with height. 


\section{MODIFICATIONS AND DISCUSSION}

The photospheric quantities that can be measured with a vector magnetograph at present are the longitudinal field $B_{z}$, the absolute magnitude $B_{\perp}$ of the transverse magnetic field $\mathbf{B}_{\perp}$, and the angle $\theta$ made by the direction of the vector $\mathbf{B}_{\perp}$ with a fixed direction, say the $x$-axis $\left(0 \leq \theta \leq 180^{\circ}\right)$. The transverse magnetic vector $\mathbf{B}_{\perp}$ is not completely defined, since the signs of its rectangular components

$$
B_{x}= \pm B_{\perp} \cos \theta \quad B_{y}= \pm B_{\perp} \sin \theta
$$

are not determined. What we have been looking for is a criterion to choose the correct sign in the expressions for $B_{x}$ and $B_{y}$.

In practical applications, the criterion must be expressed in terms of observable quantities $B_{z}, B_{\perp}, \theta$, and their transverse gradients. If the second term on the left hand side of inequality (5) that has been dropped in the above derivation of Krall's criterion can be transformed to fulfill this requirement, it may be retained to give a new criterion. Actually, by the solenoidal condition of the magnetic field,

$$
\frac{\partial B_{z}}{\partial z}=-\left(\frac{\partial B_{x}}{\partial x}+\frac{\partial B_{y}}{\partial y}\right)=-\nabla \cdot \mathbf{B}_{\perp}
$$

inequality (5) may be transformed into

$$
\mathbf{B}_{\perp} \cdot \nabla B_{z}-B_{z} \nabla \cdot \mathbf{B}_{\perp}<0
$$

which may be written in terms of $B_{z}, B_{\perp}$, and $\theta$ as

$$
\pm\left[\left(B_{\perp} \cos \theta \frac{\partial}{\partial x}+B_{\perp} \sin \theta \frac{\partial}{\partial y}\right) B_{z}-B_{z}\left(\frac{\partial}{\partial x} B_{\perp} \cos \theta+\frac{\partial}{\partial y} B_{\perp} \sin \theta\right)\right]<0 .
$$

Thus, we have obtained a more general criterion $(7)$ or $\left(7^{\prime}\right)$ which holds only if the magnetic lines of force are divergent and convex upward. Unlike Krall's criterion (1), the quantities to be differentiated in inequality $\left(7^{\prime}\right)$ are not only $B_{z}$, but also $B_{\perp}$ and $\theta$ which can only be measured less accurately. This is the drawback of inequality (7), since the derivatives of noisy data are even noiser in numerical calculation.

Now consider the magnetic lines of force through the photospheric plane being divergent only and drop the condition of upward convex. For convenience, the condition of upward divergence (2) may be written as

$$
B_{z} \mathbf{B} \cdot \nabla B^{2}<0 \text {. }
$$

By means of $\mathrm{B} \cdot \nabla=\mathrm{B}_{\perp} \cdot \nabla+B_{z} \partial / \partial z$ and $B^{2}=B_{\perp}^{2}+B_{z}^{2}$, inequality (8) can be expanded into

$$
B_{z}\left[\mathrm{~B}_{\perp} \cdot \nabla\left(B_{\perp}^{2}+B_{z}^{2}\right)+B_{z} \frac{\partial B_{z}^{2}}{\partial z}+B_{z} \frac{\partial B_{\perp}^{2}}{\partial z}\right]<0 .
$$

The last two terms involve the derivatives normal to the photospheric plane $\partial / \partial z$. By equation (6), the former can be transformed into derivatives of the 
transverse field in the photospheric plane. The latter may be transformed for a force-free field which may be adopted wherever the field is strong enough. From the components of the force-free equation $\nabla \times \mathbf{B}=\alpha \mathbf{B}$ on the photospheric plane:

$$
\begin{aligned}
& \frac{\partial B_{z}}{\partial y}-\frac{\partial B_{y}}{\partial z}=\alpha B_{x}, \\
& \frac{\partial B_{x}}{\partial z}-\frac{\partial B_{z}}{\partial x}=\alpha B_{y},
\end{aligned}
$$

eliminating the force-free factor $\alpha$, we have

$$
\frac{\partial B_{\perp}^{2}}{\partial 2 z}=\mathbf{B}_{\perp} \cdot \nabla B_{z}
$$

Substitute the normal derivatives in inequality (9) with equations (6) and (10),

$$
B_{z}\left[\mathbf{B}_{\perp} \cdot \nabla\left(B_{\perp}^{2}+2 B_{z}^{2}\right)-2 B_{z}^{2} \nabla \cdot \mathbf{B}_{\perp}\right]<0,
$$

or in terms of $B_{\perp}$ and $\theta$,

$$
\pm B_{z}\left[\left(B_{\perp} \cos \theta \frac{\partial}{\partial x}+B_{\perp} \sin \theta \frac{\partial}{\partial y}\right)\left(B_{\perp}^{2}+2 B_{z}^{2}\right)-2 B_{z}^{2}\left(\frac{\partial B_{1} \cos \theta}{\partial x}+\frac{\partial B_{\perp} \sin \theta}{\partial y}\right)\right]<0 .
$$

Inequality (11) or $\left(11^{\prime}\right)$ is the criterion for a photospheric region with a strong magnetic field and upward divergent lines of force.

According to the basic magnetic field, inequality (1), (7), and (11) may be chosen as the criterion to determine the sense of the transverse magnetic field $B_{\perp}$ from the measured photospheric quantities $B_{z}, B_{\perp}$, and $\theta$ under different circumstances respectively. For various conditions, we can deduce more relations, such as the inequality (1) which holds as well for a force-free field with a transverse field decreasing with height, as it can easily be seen from equation (10). All the criteria suggested here are to be tested in practice for their feasibility.

If more physical quantities other than the photospheric $B_{z}, B_{\perp}$, and $\theta$ can be measured, new criteria may be derived to solve the $180^{\circ}$ ambiguity in the direction of $B_{\perp}$. For instance, recently the longitudinal magnetic field in the chromosphere can be measured using the $\mathrm{H} \beta$ spectral line with the solar magnetographic telescope at the Huairou Station of the Beijing Observatory. A new criterion has been suggested using coordinated observations of photospheric and chromospheric magnetic fields (Wu \& Ai 1990).

\section{ACKNOWLEDGMENTS}

The author wishes to thank Dr. Wang Jinxiu for helpful discussions.

\section{REFERENCES}

Krall, K.R., Smith, J.B., Hagyard, M.J., West, E.A., \& Cummings, N.P. 1982, Solar Phys., 79, 59

Wu, L. \& Ai, G. 1990, Acta Astrophys. Sinica, 10, No. 4, 371 